\title{
LA MONDIALISATION ET LA CRISE EN DROIT : REPENSER L'ÉTHIQUE ET LA MORALE DANS L'ÉTAT POSTMODERNE
}

DENDASCK, Carla ${ }^{1}$

\section{SILVA, Adoniya Osias ${ }^{2}$}

DENDASCK, Carla ; SILVA, Adonijah Osias-la mondialisation et la crise en droit : repenser l'éthique et la morale dans l'état post-moderne - connaissances scientifiques multidisciplinaires Core Magazine-Vol. 1. An. 1. Mars. 2016, pp: 98-119ISSN: 0959-2448

\section{RÉSUMÉ}

Ce test a pour but une analyse critique sur la mondialisation économique et technologique et son impact sur le droit positif, avec sa logique de normativista visage formelle les contextes qui sont de plus en plus complexe et changeant. A partir de cette prémisse, il est prévu d'évaluer comment l'éthique et la morale peut servir du Nord dans ce changement de paradigme qui est nécessaire pour une application de la loi qui répond le mieux aux aspirations du citoyen dans une société en constante mutation. Le but est d'analyser le rôle du droit en vertu de ce nouveau biais de mondialisation et de son importance pour l'homme moderne.

Mots clés : éthique. Moral. Mondialisation. Droit

1 Doctorat en psychanalyse, doctorant en psychanalyse clinique, maîtrise en bioéthique-directeur du centre de recherche et d'études avancée-email : prof.dra.csd@hotmail.com.

${ }^{2}$ Avocat, études supérieures en droit fiscal ; Maîtrise en droit à l'Escola Paulista de Direito ; Adonias.Silva@UNIFESP.br. 


\section{INTRODUCTION}

Est sabença générale que la trajectoire de l'homme sur la planète, commençant dans les cavernes jusqu'à l'homo sapiens, quand l'homme a commencé à prendre conscience de votre intelligence et de la valeur vers le haut de sa liberté pour surmonter les obstacles qui toujours a croisé son chemin, a toujours été marquée par la participation à la construction du droit et la justice.

Même à l'aube de la civilisation, quelle n'avait pas une idée d'amélioration droit et la justice comme nous ont de nos jours, l'homme a toujours lutté pour être que l'acteur de sa propre histoire et au sein de ce morceau est devenu un politique et morale, qui a agi dans son concept d'éthique et de moralité.

En effet, on distingue l'homme des autres êtres vivants exactement pour son développement intellectuel, qui à son tour est directement liée à sa conduite dans la société, c'est-à-dire dans son intérieur moral est démontrée par le biais de leurs attitudes sur la coexistence collective avec leurs pairs, qui est ne pas le cas avec l'autre vivant des êtres qui agissent simplement par instinct. Que sa position parmi les autres machines se déplace de la nature est qui donne une place de choix comme social Dominator (REALE, 1994).

Depuis les coutumes sociales transmis de père en fils les relations entre les groupes sociaux ont évolué à partir des paradigmes simples coexistence jusqu'à ce que la structure de commandement plus solide tels que le droit de positivado et que par suite de l'éminemment sociable de l'être humain de leur coexistence sociale fait exsurgir le droit, comme un phénomène social de leur propre culture avec ses règles et sanctions.

Et à ratifier cette réalité, il existe un principe Latin qui dit: « ubi societas, ibi ius est "(où il y a la société, il y aura un droit). C'est le point de départ de cette épreuve, puisque la société a évolué, il est devenu mondial et le droit positif avec ses règles formelles, semble-t-il, peut ne répondent plus aux aspirations de cet homme moderne qui, dans un monde globalisé, suit dans son voyage éternel dans la poursuite de la Justice. 
Mondialisation, qui est venu main dans la main avec la révolution technologique et les changements économiques, après avoir remporté sa première phase d'installation, qui est l'intégration des marchés mondiaux, nous présente maintenant un nouveau défi sont les ramifications et les répercussions sur les institutions juridiques. Le grand défi est de savoir qui l'efficacité et la portée des institutions juridiques et des instruments juridiques pour cette nouvelle réalité de l'homme globalisé.

A côté de cela, vous ne peut pas oublier le rôle de l'éthique et la morale du droit moderne, fondations pour une société juste et correcte. Un tel fait peut être vu par le simple fait que, dans la pratique, l'application pratique de la loi se faite par l'intermédiaire de la justice distribuée pour un homme - les juges - qui le système juridique se trouve avec le mister honorable pour résoudre le différend entre les parties au différend, à la recherche d'une phrase.

En comparant le processus procédural à la recherche de la phrase ce que vous vous rendez compte, c'est qu'ils sont placés dans la confrontation non seulement légale, mais à tous les principes éthiques et moraux que les dispositions de procédure imposent non seulement les parties au litige mais aussi son propre juge. Le point d'achoppement est de savoir comment être éthiques dans la corruption dégénérée, intentée au sein de la mondialisation et leurs systèmes technologiques, où vous pouvez détourner d'un simple choix du liquidateur de condo jusqu'à une élection pour choisir nos dirigeants.

Mondialisation économique impose le devoir de l'homme alors Rico, d'avoir, de posséder à tout prix et des innovations technologiques sont les pistes qui conduiront à la locomotive de la malhonnêteté, sous prétexte d'aller que la fin justifie les moyens, plus loin, l'homme des valeurs éthiques et morales provenant du berceau. Et dans ce système la nexted «Loi de Gerson », de la facilité à tout prix, est la Loi, avec tous ses aspects et facteurs déterminants.

II s'agit de la crise dans le droit d'être traiter dans ce texte, en tenant compte des aspects de la mondialisation, qui a fait du monde un village planétaire et prit avec lui les valeurs de l'éthique et la morale transmise à nous par nos pères fondateurs. Nous 
vivons aujourd'hui dans des contextes de plus en plus complexes et mutables, qui clameur pour une loi contextualisée, une loi qui répond aux besoins actuels de l'homme, qui ne sont plus le même homme inséré dans le contexte du siècle dernier.

Que peut-on dire du béton est que l'État et le droit, modelée sur nous sommes déployés dans la société aujourd'hui, ne pas adapté à l'évolution sociale vécue par les humains dans la société traditionnelle. Aujourd'hui, plus que jamais, le droit doit prendre d'autres directions si vous voulez répondre aux aspirations de cet homme. Le droit doit se tourner vers des moyens alternatifs, c'est-à-dire, la Loi doit être un droit de négociation, de tables rondes, d'arbitrage et de médiation.

Ce qui est certain, c'est que les chemins qui nous a apporté cela jusqu'à maintenant ne sera pas le même qui nous mènera à l'avenir. Et à cause de cette réalité est valide d'analyser dans quelle mesure la positivado droite, avec une logique formelle et rigide, peut répondre à la satisfaction les aspirations de cet homme globalisé, en particulier en ce qui concerne l'aspect éthique et moral. N'est pas nécessaire de maintenir l'éthique et la morale sans perdre de vue la Loi et son application correcte au cas particulier.

\section{MONDIALISATION DANS L'ÉTAT MODERNE}

L'histoire a montré que l'homme a toujours lutté pour l'amélioration de leurs conditions de vie. Conglomérats humaines a déjà pris la forme des villes vers 3500 av. J.-C. et son contexte social ont émergé les guerriers avec leur primitif armes ont été forgés pour la défense du groupe social. Cela a donné naissance les constructeurs de son toit mansardé, qui a dominé l'utilisation de l'eau, et ceux qui, avec sa sagesse, a appris à interpréter les phénomènes naturels et sont devenus leurs prêtres et les juges.

De ce développement primaire homme et de la société a évolué de différentes façons et à plusieurs étapes, que vous ne voulez pas eux, analyse ici depuis cet article ne se prête pas à ce souhait. Toutefois, il est intéressant de noter ici seulement les abolutismos qui ont marqué les débuts de l'État moderne, qui a comme caractéristique le monopole de l'usage de la violence par les despotes, que le moyen absolu détenait 
le pouvoir à tout prix, n'étant ne pas seulement des législateurs, mais aussi juger de leur propre volonté.

L'État, afin de maintenir la paix sociale menacé ou violés, a interdit l'autotutela et appelé à la responsabilité de régler les différends. Partir de ce moment, l'État a désormais la fonction de l'application de la loi aux cas spécifiques soumis à elle et fixer le conflit.

Ainsi l'État comme titulaire de l'ordre, le droit et la justice en limitant le champ des souverains à des limites strictes de la Loi, donnant le pouvoir judiciaire de la compétence exclusive pour juger et régler les conflits et ce fait par le biais de la compétence. La compétence de mot vient de la composition de deux mots : le substantif Latin jus, juris, ce qui signifie « droit », plus la troisième conjugaison du verbe dicere, qui signifie "dire", "dire", "express". Ainsi, la compétence est le pouvoir que l'État a le droit de dire.

Cependant, la compétence est inerte, le principe du Latin: "nemo iudex t et sine ; NE procedat iudex t Office (aucun juge sans acteur ; le juge doit d'Office). C'est pourquoi l'art. 2 du code de procédure civile, qui donc prolata: « aucun juge ne fournira la protection judiciaire sinon comme partie intégrante ou intéressée à appliquer, dans les cas et les voies légales ».

Pour cette raison, afin que l'État peut offrir les performances et exercer son rôle comme un médiateur des litiges n'est pas nécessaire que le titulaire du concurrent les intérêts dans la relation juridique de matières de droit a l'initiative et aller frapper aux portes de la magistrature. Par lieu, vaut de noter que bien que l'État n'a pas la compétence pour commencer, une fois causé la fonction juridictionnelle de l'État, le processus sera développé par officiel d'impulsion jusqu'à sa finalisation avec une phrase qui a mis fin à la transaction.

Avec la croissance démographique et la croissance économique, technologique et sociale, la demande des citoyens touchant aux portes de la magistrature sont proportionnellement plus grand que les réponses données à ceux qui cherchent une 
réponse à leurs exigences et leurs différends. Et le résultat de ceci est que le pouvoir judiciaire, qui ne répond plus aux exigences il pris, un activisme judiciaire qui ne correspond pas aux préoccupations des citoyens.

Aujourd'hui, avec la mondialisation, les problèmes rencontrés par la société sont complètement différentes de celles vécues par la génération précédente et la tendance est à une augmentation de ces difficultés, et la certitude que nous avons est que le pouvoir judiciaire n'est pas prête pour les changements qui se présentent à la prochaine génération, dont nous parlerons dans les lignes suivantes.

Quand il s'agit de ce que la mondialisation a en tête est une analyse sérieuse des processus et liés entre eux et les changements qui sont produisent sur la scène mondiale et avec elle tout le chemin de penser, agir et vivre dans la société évolue considérablement. Ces modifications affectent non seulement les structures économiques et sociales qui interfèrent directement en production juridique actuelle et, par conséquent, sous la forme de penser et de faire du droit.

Le premier point que vous ne peut pas ne pas mentionner est la question de la souveraineté de l'État-nation face à la croissance de multinacionalismo et transnationalisme. Avec l'avènement de sociétés multinationales et l'émergence de rampantes supranationales et transnationales agents, porté par la mondialisation, l'État-nation voit sa force mitigé en ce qui concerne le règlement et structuration des régulations économiques, ainsi en ce qui concerne la protection de l'individu et la société. Ce qui était autrefois une compétence exclusive de l'État-nation, de la mondialisation sera la cible et l'intérêt des autres institutions transnationales et multinationales.

Les entreprises multinationales, avec leur capacité d'expansion, de production et autres opérations dans le monde, en dépit de son importance pour le développement social et économique, imposent le droit d'une façon complètement différente de penser mis moules du siècle passé et qui a été perpétuée dans l'histoire. La facilité avec qui ces entreprises doivent déplacer leurs usines d'un pays à l'autre ainsi que leur potentiel 
monde trading, exigeant le droit un aperçu et plus ouverte et moins systématique et fermé, à proximité de l'actuel.

La pertinence de ces entreprises pour l'économie mondiale a conduit à un changement d'orientation à des relations sociales, avec toute sa complexité, exigent l'avocat non seulement le point de vue national, cependant, un look 'au delà des frontières", c'està-dire un regard global. Même qu'est devenu un lieu commun si vous entendez que « le monde est un village global, » ce qui signifie que les clôtures, murs et les frontières qui nous séparent de nos voisins, maintenant ne peuvent plus être entrevu.

Cette réalité porte atteinte au droit à tous les niveaux et a ses développements dans des valeurs éthiques, moral et bon comportement requis du citoyen est entré dans ce contexte social mondialisé. Exposé à tous les risques engendrés par la mondialisation, ce citoyen, qu'une réponse de la demande en justice à votre demande, vous ne trouvez pas le droit plus que des concepts et instituts, a officiellement établi et déterminé, qui ne peut pas répondre à vos besoins au sein de cette complexité globale.

Pour cette raison, nous pouvons dire que les points de la mondialisation à un changement dans les affaires civiles, pénales, administratives et, surtout, le droit international, vous devez adapter constamment pour répondre à des situations nouvelles et inhabituelles qui jusqu'à ce qu'il ya quelque temps étaient censés existent, tels que le commerce mondial et financier commercialise I'Union qui, malgré sa flexibilité pour créer la capitale unie à l'échelle mondiale et de la facilitation de la libre circulation des investissements sans frontières, une nouvelle réalité et diffuse à la législation en vigueur.

Un autre facteur qui doit être considéré dans ce monde globalisé est la question de la croissance des blocs économiques régionaux, car il affecte directement le modus operandi du droit tel qu'il est dans notre société. Ce qui est la plus répandue aujourd'hui et qui a augmenté chaque année, est l'échange entre les blocs commerciaux régionaux, à savoir la réduction des obstacles au commerce entre les Nations. N'oubliez pas de sigles comme la ZLEA, MERCOSUR, UEA, juste pour en nommer quelques uns de ces blocs qui relient avec affinités commerciales. Ce qui est certain, 
c'est que le commerce international est aujourd'hui une réalité et les concepts néolibérales qui étaient plus hégémonique, ouvrent les portes de nombreuses nations anciennement fermées au dialogue, comme c'est le cas en Europe de l'est, l'ancienne Union soviétique et, avec des réflexions positives sur la Chine.

Le libre-échange international et l'accent exacerbé que les marchés privés en quelque sorte décentralise et réduit le rôle du gouvernement, qui a toujours été l'organisme de réglementation de l'économie. Cette modification des échanges libres de ces manèges directement influencé la politique économique de «Oncle Sam », ainsi que la politique économique européenne en général, conduisant à la création d'entités telles que le FMI, la Bird (ainsi que les banques régionales et le GATT), qui s'étend ses effets dans le monde globalisé.

Et si tous ces changements ne suffisait pas pour tracer le graphique de la société actuelle, vous pouvez toujours parler sur la tendance mondiale à la démocratisation, la protection des droits de l'homme, prenant une participation beaucoup plus grande dans le domaine du droit, compte tenu de la fonction juridictionnelle de l'État pointé au départ. Parallèlement, les manifestations flagrantes par les changements économiques, un effort considérable au niveau international, pour la création de politiques libérales, qui visent à protéger les droits de la personne, ainsi que le contrôle de l'arbitrage et le renforcement des institutions juridiques qui demande vraiment judiciaire viennent de fournir aux citoyens, visà-vis de ses intérêts diffus et collectifs.

Parallèlement à tout cela vous y trouverez également les ONG qui sont des acteurs transnationaux et supranationales qui apparaissent dans ce scénario afin de protéger les droits de l'homme, les droits des plus démunis, le droit à un environnement durable, les droits des peuples autochtones, les droits des minorités ethniques, la question de l'affectif dans la société, homo n'en citer que quelques unes. Vous ne pouvez pas oublier que toutes ces questions font partie de la vie quotidienne du citoyen et la société attend avec impatience une réponse satisfaisante et vous venez de trouver qu'homme moderne qui, semble-t-il, a été prié de quitter la grotte, n'a cependant pas eu les moyens d'avancer, c'est pourquoi encore absorbé à tout ce que vous voyez 
autour de vous se sentir impuissante et mal préparés pour faire face à cette nouvelle réalité.

D'analyser tout ce problème mondial est le droit des brevets s'est efforcée de plomberie et de conduite des conflits et des affrontements survenus dans la société, c'est pourquoi il faudrait parler non seulement à un radical et urgente, à une remise en forme totale des concepts traditionnellement utilisé, en manière que, de la même manière que l'homme est sorti de la grotte le droit aussi faire de la même façon et venons de constater que l'homme moderne au milieu d'une société chaotique, un monde globalisé et une expérience des conflits généralisées à tous les niveaux.

\section{POSITIVISTE DROIT ET LOGIQUE FORMELLE}

II n'est pas possible de parler au positivisme dans domaine juridique sans remorque à la figure de Hans Kelsen. En effet, bien qu'il est considéré comme le père du positivisme convient de souligner que tout ab initio théorie positiviste se construisait au cours des années et avait son rôle de premier plan dans le développement du droit comme une science.

Pour Kelsen, la Loi doit être comprise comme norma, divorcée de n'importe quelle conception sociale ou évaluative. II suffit de penser de ce mode est la pièce maîtresse de son œuvre, appelée théorie pure du droit, la libération de cette science juridique à tout le contenu, de l'optique de Kelsen, pas son propre. En proposant ce clivage du juriste allemand vise à donner une science d'autonomie juridique propre, exempte d'éléments qui n'appartiennent pas à eux.

Pour Kelsen science juridique pure doit être sans équivoque à la psychologie, la sociologie, politique et morale. Bien que ces Sciences ont trait d'une certaine façon à droite et traversent votre chemin la plupart du temps, et pourtant la science juridique ne peut pas être imprégnée de ces concepts en dehors de son domaine d'expertise. Cette compréhension, même si cela semble étrange à l'oreille, a sa valeur lorsque vous voulez parler de la justice, comme si en utilisant un langage précis et rigoureusement logique, Kelsen abstrait bon concept l'idée de Justice. 
La justice est toujours et invariablement étroitement liée avec les valeurs (toujours variables) adoptées par celui qui l'invoque (que ce soit par des arguments sociologique, psychologique ou morale), n'est ne pas une notion de droit universellement valable, en raison de l'imprécision et la fluidité du sens, faire ce qui est bon pour un contexte donné ou la société qui ne serait pas juste de l'autre.

Dans la vision de Joram, le droit est limité à un ensemble de règles représentées par la catégorie des exigences « devrait être » et apporte ainsi les faits à une échelle légale ou antijurídico. Avec qui on s'aperçoit clairement que Kelsen crée une éthique fondée sur la distinction entre être et devrait être. En d'autres termes, Kelsen établit une distinction entre la façon dont les choses sont et les façon dont les choses devraient être et cette relation entre " être » et le "must-être" se termine par deux aspects distincts, et qui sont indispensables dans sa conception. Le premier de ces aspects concerne le fait que cette distinction entre l'être et doit-être sert à différencier les deux façons dont on peut étudier la Loi : la première est la vision du droit tel qu'il est, et le second est la vue de la Loi comme il se doit. Le deuxième aspect que cette distinction sert aussi à faire un point de repère qui sème la discorde entre le Royaume des faits liés à l'être- et le Royaume des normes-devraient s'enchaîner. Par conséquent, l'équation suivante : le défaut de se conformer à l'obligation (l'absence de fait donné), implique la sanction (devrait être). En termes plus clairs : l'échec (devoir), il devrait y avoir une sanction (sanction établie par la norme).

Dans ce contexte, Kelsen note que la norme est un produit de la volonté, élaboré dans le but de réglementer le comportement humain et qui sert comme une interprétation. Ici, il est nécessaire de souligner que pour le positivisme à la norme n'a aucune validité pour le sentiment d'être juste, mais pour le simple fait d'être connecté à une autre norme considérée comme supérieure, il a appelé la norme fondamentale. II ressort de ce concept méthodologique de norme fondamentale qui le juriste peut créer une systématisation d'un ordre juridique entière.

Un de ses concepts théoriques pratiques plus loin est l'idée du système juridique comme étant un ensemble hiérarchisé de règles juridiques structurées sous la forme d'une pyramide abstraite, ponctuée et dominée par la Constitution de l'État, qui rend 
les autres règles juridiques de hiérarchie inférieure (les différentes lois infraconstitutionnelles et autres actes normatifs). Cette conception théorique est que des extraits la notion de rigidité constitutionnelle, qui est activer et exiger un système pour protéger l'intégrité de la Constitution. Malgré les controverses sur la création de la pyramide normative, dans le but de cet essai est attribué à Kelsen.

Une autre contribution majeure du juriste au monde pratique du droit a été la Constitution de l'Autriche de 1920, élaboré dans le cadre de son inspiration. Dans l'ombre de l'influence de Kelseniano pensé que cette lettre politique autrichienne, marketing aux précédente droit positif innové le concept du concentré contrôle de constitutionnalité des lois et normatif agit comme une fonction judiciaire par une Cour constitutionnelle, chargée de la fonction exclusive de l'intégrité de la Constitution.

A partir de là, la juridiction constitutionnelle peut être divisée en deux parties : la juridiction constitutionnelle (concentré contrôle de constitutionnalité) et diffuse la juridiction constitutionnelle (contrôle diffus de constitutionnalité). Ce dernier mode de garde de la Constitution (diffuse) était déjà pratiqué dans les États-Unis d'Amérique.

Dans le système juridique brésilien, sous l'égide de la Constitution fédérale de 1988, la juridiction constitutionnelle est pratiquée de deux façons : le concentré, par des actions de la compétence de la Cour suprême et la diffuse, exécuté dans les comptes rendus des actions (et ressources à ces inhérente) relevant de la compétence d'un tribunal, si juges ou tribunaux.

Par conséquent, lorsqu'il est question de Kelsen nous ne pouvons pas perdre de l'essentiel de sa pensée qui est la validité de l'état de droit et de leur hiérarchie, étant l'un des aspects plus importants de sa théorie.

Avant que le plus haut qu'on peut voir que la loi positiviste est un ensemble de règles qui régissent la conduite humaine et ces normes, à son tour, lors de l'échec valide et légitimement perçu acquièrent un pouvoir d'établir des sanctions, en raison de sa structure, se caractérise comme un ordre coercitif et qui s'applique uniquement si vous sont indissociablement liées aux autres standards. 
Le gros problème qui se présente en ce qui concerne le positivisme juridique, c'est que même si il s'agit d'un système de règles régissant la conduite de l'homme dans la société, mais la rigidité de le positivado droit ne peut pas répondre aux préoccupations de ce même homme. Ce tapis roulant est utile de mentionner les critiques formulées par Dworkin au positivisme juridique en disant que le positivisme est tellement dogmatique que «la loi devienne souvent ce que dit le juge » (DWORKIN, 2003, p. 4). Cette critique de Ronald Dworkin fait contrepoint à la loi positiviste montrant un modèle théorique qui a une étendue pour donner une réponse adéquate à la question de l'interprétation, qu'il appelle l'intégrité du droit

Eh bien, ce dogmatisme intolérant du positivisme juridique, qui en fin de compte réduire la Loi de l'instruction de certains juges qui est en prenant l'espoir des citoyens, reléguant le droit seulement pour le monde des idées, ce qui devrait être et pas le monde réel, ce que l'on attend quand il est entré dans la magistrature avec une demande : le concret de l'action en justice. Ne parlez pas ici dans la justice, parce que, comme cela a été mentionné, le positivisme juridique n'a pas que la revendication en justice, mais vise simplement à fournir un concept universellement valide du droit, en dehors d'autres réalités sociales. Le point ici est qu'au moins l'entrent en vigueur les dispositions juridictionnelles tellement envie en fait et en droit et non seulement sousjacents dans les dispositions légales, verbalisé par toga.

\section{ÉTHIQUE ET LA MORALE DANS LE DROIT}

Quand il s'agit de l'éthique et la morale dans le droit de ne pas oublier que le comportement d'un individu par rapport à l'autre, dans tous les contextes sociaux dans des combats judiciaires, menées à la justice, a toujours une forte inclinaison morale.

La seule différence entre les postulats éthiques et morales et du droit est que ce dernier a sa force coercitive, régi par le système judiciaire, que toutes superposées avec ses règles et normes définies, qui dicte apporte avec eux les peines responsables à qui ils soumettent. 
Le juriste positiviste Hans Kelsen (2001:36), déjà mentionné dans ce texte, apporte une contribution importante à la compréhension de ce thème éthique et la morale dans le droit. Dans son ouvrage " qu'est la justice », le savant allemand est ainsi exprimé :

Tout en reconnaissant le droit comme la technique coercitive spécifique de l'ordre social, nous pouvons le comparer avec les autres ordres sociaux qui, en partie, ils poursuivent les mêmes objectifs que la Loi, mais par des moyens différents. La Loi est milieu social spécifique, non une fin. La Loi, la morale et la religion - les trois interdisant meurtre. Mais la loi ne prévoyant donc que : si un homme commet un meurtre, un autre homme, désigné par la Loi, s'applique contre le tueur, une certaine contrainte prescrites par l'ordre juridique. La morale exige simplement : tu ne tueras pas.

Il convient de noter que bien que le moral n'a pas la puissance et la force à mettre en place une sanction pour leur désobéissance, comme le fait la droite, encore il ne peut pas être négligé par les spécialistes du droit, depuis avant même le positivado droite avec leurs mœurs et des concepts déjà a été utilisé par les sociétés primitives comme un moyen pour orienter et diriger les relations dans la convivialité sociale.

Par conséquent, la distinction entre le principe moral et juridique est une ligne fine et ils vont souvent de pair et suivant et qui sont séparés seulement par ses effets. Laissez-nous prendre comme exemple un fils que lors du passage son père et reçoit un compliment ne pas la pareille la même courtoisie, ou même distrait, un tel comportement est une offense morale pour laquelle il existe une douleur morale. Toutefois, si ce même fils, pour être accueilli par son père provoque un préjudice corporel, en l'occurrence commet le crime prévue dans la planification et doit être puni.

Pour cette raison, Chaïm Perelman (1996:289), le grand philosophe de Bruxelles et l'un des plus grands philosophes du XXe siècle. XX, d'aborder ce thème et la loi morale, laissé inscrit ce qui suit :

Traditionnellement, les études consacrées aux relations entre le droit et la morale insistent, dans un esprit de Kantian, en ce que le distingué : le droit régit le comportement ; la morale met l'accent sur l'intention, la Loi établit une corrélation entre 
les droits et obligations, les devoirs moraux prescrit qui ne donnent pas lieu à des droits subjectifs, la Loi établit les obligations sanctionnées par la puissance ; la morale de sanctions organisée s'échappe. Avocats, insatisfaits d'une conception positiviste, des statistiques et formaliste, insistent sur l'importance de l'élément moral dans l'application de la Loi, dans le rôle qu'ils jouent de la bonne foi, mauvaise foi, une intention malveillante, moralité et tant d'autres notions dont aspect éthique ne peut être bafouée.

Par les mots du philosophe, érudit de Bruxelles peut déjà avoir une idée de l'ampleur que la morale et l'éthique joue dans l'application du droit à nos jours. Nous vivons dans une société où l'éthique et moral valeurs confondre avec l'intérêt personnel et privé, ce qui explique pourquoi ces valeurs sont plus en plus reléguées à l'arrière-plan, caractéristiques d'une société de consommation, où ce qui importe est la réalisation des objectifs, quels que soient les moyens utilisés pour faire, faire le vieux principe prévaut que la fin justifie les moyens.

Parce que c'est exactement dans cette ligne fine entre les valeurs morales et éthiques et de la morale que le droit doit marcher son chemin, une fois que la société vient de perdre ces valeurs à toutes les générations. À l'époque de nos pères, le simple mot scellé un cadre juridique et entreprise était exploitée jusqu'en des circonstances.

Actuellement, même quand ils sont entourés de toutes les garanties, les titres et tout, et pourtant vous avez l'assurance de réel que l'entreprise juridique viendra à terme de manière sont, sans l'intervention de l'État à exercer son rôle de coator nécessiter les règles initialement compactuadas.

Sur cette exigence sociale qui est imposée au droit actuel, la façon dont se matérialise dans le processus, par le biais de la décision du juge, fait apparaître l'ensemble de la collection de la culture humaine dans l'intérêt des justiciables devant la Cour de l'État, qui prend plus en plus son rôle de délégué la société coator.

Ce qui est certain, c'est qu'en comparant la pratique de la procédure sont introduits dans la confrontation non seulement des règles juridiques, mais aussi tous les 
principes éthiques qui la loi du processus exige des justiciables et même le juge luimême. Flanqué de la liberté, il y a une structure entière de loyauté et de sincérité qui est requis par la loi dans les soucis et les liens judiciaires. Ce que vous devriez obtenir n'est pas juste la norme positivada et formelle, mais aussi des valeurs éthiques et morales comme des éléments essentiels de la revendication a demandé le contrôle judiciaire.

La façon dont la société est organisée aujourd'hui, avec tous les instruments de contrôle social et de l'augmentation de l'information par le biais de la technologie et la croissance économique en raison de la mondialisation, n'est plus prête à un simple spectateur de la Justice. Au contraire, l'absence du cadre juridique a montré, ce qui est observé aujourd'hui, plus que jamais, est une société en train de prendre la loi entre leurs mains, essayant de combler le vide laissé par les autorités judiciaires sur la responsabilité judiciaire, mais malheureusement la mauvaise façon, faire, souvent sous le couvert de la Justice, de fonctionnement injustice encore plus. Tout cela pour ce formalisme juridique, qui cherche seulement à la technique et l'amélioration des lois sur les réponses aux problèmes sociaux, ce qui le rend clairement que ce modèle n'est plus supporté par la société moderne.

Comme il est à noter ici les mots du juriste italien Francesco Carnelutti (2004:60) qui a: « Si la Loi est un instrument de Justice, pas la technique ou la science assez pour y faire face. » En témoignent les paroles de l'ínclito de juriste italien l'application pratique du droit, ce que vous faites par la Justice menée dans les conflits juridiques, exige plus que des techniques juridiques et des normes de gestion de la justice. La grande question que vous ne pouvez pas cacher est que l'application directe comme il est fait de ces jours, le positivado, le prêt et le formel, visa vraiment justice. On peut voir que l'application des problèmes pratiques portées à l'appareil judiciaire ne sont pas répondre avant les concepts juridiques préétablis et strictement formel.

II n'était pas sans raison que Jhering (2012:322) a déclaré que les problèmes pratiques ne s'adaptent pas bien au que paradis des concepts en ce que bon nombre d'avocats préfère vivre. Selon João Maurício Adeodato (2012:317), a proposé une interprétation 
moins dogmatique de Jhering, auteur qui est internationalement considéré comme l'un des plus importants pour la dogmatique du droit privé.

Étant donné qu'on se rend compte que la Loi doit marcher à la recherche d'un équilibre entre le normativista de vision positiviste de Hans Kelsen, où la décision du juge doit être façonnée par la posta (droit donc positif) et la position plus extrême

proposé par le Viehweg topique, pour lequel la décision ne devrait pas garder relation avec les textes réglementaires. À cet égard, João Maurício Adeodato (2012:313) écrit avec beaucoup de propriété sur le thème :

Le Viehweg topique semble conduire à une excessive par rapport au texte réglementaire, qui est considéré comme seulement un dessus d'expression parmi d'autres « ouvrir ». En refusant le lien nécessaire de postulat avec texte dogmatique, topique fait le « orientation par le biais de » une méthode trop libre et avoisine les pouvoirs de décision.

Que se passe-t-il si les tentatives de sauvetage est ce dogmatisme du droit positif qui recherche uniquement et alors que la norme fixée par l'État leur soutien base, au détriment des arguments moraux et éthiques dans la recherche de la justice. Ce qui est plus important est le système juridique actuel, estatalmente positivado et régis par leurs propres règles très formelles.

Comme un corollaire de ce décor formel et rigide, ce qui n'est plus conforme aux souhaits de l'homme moderne est que les autres moyens de règlement des différends, comme la médiation, arbitrage, conciliation, qui se font passer pour des compléments à la magistrature en essayant de le faire. Ces instituts apportent au milieu de toi non seulement une alternative à la traditionnelle façon de résoudre les conflits, mais une recherche et de sauvetage par les valeurs morales et éthiques qui se manifestent dans l'action des médiateurs et des arbitres.

Dans le scénario de ces nouveaux instituts qui se posent dans notre société est qui doit briller comme un comportement professionnel de l'avocat, tester son éthique et la position morale face aux chocs qui apparaissent argument de plus en plus 
controversée. A d'autre part le droit du client qui parraine ; d'autre part, comme un adversaire, la réponse de l'autre partie, qui a aussi son avocat patron.

C'est dans ce contexte que l'avocat doit exercer leur tâche ardue en intellectuel de la défense de votre client, cependant, est restée debout et conformément à sa croyance dans le droit de se défendre et qui cherchent la justice, sans oublie les valeurs éthiques et morales qui a mené sa formation à l'heure actuelle, c.-à-d. tous votre éthique et moral du patrimoine qui a été créé au cours de sa carrière terrestre.

\section{ÉTHIQUE ET LA MORALE DANS LA RÉALISATION DE LA JUSTICE}

Une fois analysé la relation entre l'éthique et la morale, la Loi est adresse valide le revers de la médaille, qui est de l'éthique et la morale dans l'exercice de la Justice. Si la Loi de positivado, comme l'est aujourd'hui, recherche uniquement la norme contrôlée par l'Etat et strictement formelle, sans prendre en compte les valeurs éthiques et morales et la morale établie par la société, il convient de faire une réflexion sérieuse sur la contribution de l'éthique et la morale dans la réalisation de la Justice.

II est connu que l'objet est morale éthique. La moralité de mot dérive du Latin, signification "douanes ", qui, à son tour, est la partie de la science qui s'occupe du bien, la morale essentielle à la vie sociale de l'homme. Est l'ensemble des règles pour la conduite humaine comme vertu. Ainsi, la morale est indispensable pour le contenu du droit, surtout quand il s'agit de la mise en œuvre de la disposition compétence, qui a lieu au moyen de la Justice.

La science des devoirs est connue comme l'éthique et les principes axiológicos qui règlent leur, ne peut se passer d'éthique. Selon l'éminent juriste Miguel Reale: « éthique est la science des critères normatifs ». Seulement l'être humain détient ce legs, qui diffère des autres créatures vivantes, comme déjà mentionnés au début de ce texte.

Est valide ici les paroles de José Renato Nalini (1997:30), qui est connu comme l'un des plus importants théoriciens de l'étude de l'éthique au Brésil, qui exprime : 
L'éthique est la science du comportement moral des hommes dans la société. C'est une science parce qu'il a objet lui-même, ses propres lois et la méthode brevetée. L'objet de l'éthique morale. La morale est un des aspects du comportement humain. L'expression provient les mœurs romaines Word avec un sens de manières, ensemble de normes acquise par l'habitude a réitéré de sa pratique.

Par conséquent, on peut supposer que l'éthique est la science qui étudie les actes de la conduite humaine dans le contexte social fondé sur la loyauté et de la décence, en douceur des actes et la dignité de la posture, afin que la recherche de la vérité est la plus grande cible à atteindre, plus personnels et les intérêts particuliers des passions. Malheureusement, ce qui est observé est que les intérêts personnels ont surmonté les valeurs morales et éthiques et la réelle volonté de la Justice, et c'est cette situation qui ne peut se maintenir que dans cette mondialisation et société de l'information.

Ce qui ne faut pas oublier, c'est que l'éthique devienne, dans ce contexte actuel, une condition sine qua non pour vivre une vie droite dans cette société mondialisée. Sans éthique est insupportable la vie en société et de loyauté, qui est sa principale vertu, devrait guider non seulement la relation, mais afin de constituer le noyau de son essence même.

II n'est pas sans raison que l'étude de l'éthique vient au fil des millénaires et les âges, et c'était source de préoccupation pour la Grèce antique et l'érudition classique, dont les contributions soient considérées jusqu'à aujourd'hui quand il s'agit d'éthique et de morale thème.

Vous pouvez, juste à titre d'exemple, le nom Aristóteles9, qui a reconnu dès un jeune âge comme un disciple de Platon (qui, à 41 ans, a été nommé par Philippe de Macédoine, précepteur d'Alexandre le grand) et une source inépuisable de sagesse humaine, dédiée à son fils de Nicomaque, né de son second mariage de Herpile de Stageira, un de ses ouvrages sur l'éthique qui encore aujourd'hui est publiée dans toutes les régions du monde. 
Selon Robison Barone (2012:23), dans le Guide de déontologie de son avocat, cite l'estagirista comme suit: «... ce n'est pas une étude théorique que les autres, parce que nous avons étudié pour ne pas savoir ce qu'est la vertu, mais pour être bon, qui autrement serait ne vous aucun bien "(livre Il de l'éthique à Nicomaque).

Pourtant, Miguel Reale (1994:24), un autre juriste important de la scène nationale, enseigne que le huitième livre de l'éthique à Nicomaque, Aristote, développe sa théorie de la Justice et il dit :

... l'adjectif de concurrence déloyale s'applique aux deux types de personnes: 1) pour ceux qui désobéissent à la Loi ; 2) pour ceux qui veulent recevoir plus que leur part, c'est-à-dire la partie qui, par la loi qu'ils devraient être accordés. En revanche, la foire est ce qui obéit à la Loi et ce qui est content de sa part.

Ne pouvait pas aider mais n'oubliez pas les illustres Baruch Spinoza (2010), ou Bendictus Spinoza, comme il l'a lui-même utilisé pour signer dans ses écrits, qui gênaient également avec ce thème de l'éthique, développant un style hermétique, puisque la définition de Dieu, à travers les sentiers à la recherche de la liberté de l'âme de la puissance de la raison.

II y a donc, par les paroles de ces penseurs nobles, que l'éthique a son rôle prédominant dans la mise en œuvre de la Loi et dans la quête de justice. En théorie aussi, dissocié de pratique a entravé et frappé au cours de la justice au cours des années. II convient de réitérer ce qui a été dit sur la complexité des enjeux qui émergent dans cette société mondialisée.

Ce qui est certain c'est que l'histoire mondiale est écrit à une vitesse vertigineuse et accélérée, à la différence des générations passées, où les avances ont reçu les pas lents et avaient de bonnes conditions dans une certaine mesure, à se soutenir et de répondre adéquatement aux préoccupations des citoyens.

Aujourd'hui, avec l'avancement de la science, les nouvelles découvertes dans le domaine génétique, miracles et effrayant croissance internet vie générée dans les laboratoires, le clonage d'êtres humains, la longévité de la vie, le flot de nouvelles, la 
fascination de l'ordinateur et l'épidémie de cellulaires, l'homme moderne n'a plus la même certitude que leurs ancêtres. Maintenant, tout est relatif : le transmuda aujourd'hui sur hier, à la vitesse d'un atome.

Dans que tout développement et technologie de pointe, la seule chose que l'homme n'a pas, jusqu'à présent, a été la formule magique de la solidarité, le respect de l'autre homme, pas le prédateur de la nature de pires et les plus cruelles. Malheureusement, de désenchantement cette humanité, qui enchante avec une technologie de pointe, les grandes découvertes et les progrès de la science, la terreur est devenu la position éthique de l'autre, qui agit violemment comme un animal stupide, pas seulement à l'étranger, mais également la loi usurpant si rage contre tous ceux qui veulent protéger ces intérêts.

Dans ce contexte, il est entendu que la morale et l'éthique est des acteurs incontournables pour le sauvetage de cette société corrompue et avide de droit et la justice. Aujourd'hui, plus que jamais, il y a un besoin urgent de réhabiliter l'éthique de notre société afin de ramener l'homme à sa dignité. Les voies de la violence, l'égoïsme, orgueil et l'indifférence pour ses semblables, sont les fondements de la perte de morale et d'éthique. II ne suffit pas de reconnaître la dignité de la personne humaine dans l'acte normatif si le comportement personnel expérimenté dans la société a été guidé par lui.

Ce qui est certain c'est que l'éthique et la morale, une fois rachetée, sera propulseurs et pilotes vers un avenir d'espoir et de promesses pour l'humanité, qui bien que face à tant de technologie et de développement, a transformé les pratiques mesquines et primaire, que les hommes plus loin de convivialité sociale qui intègrent. C'est le grand défi pour les sociétés modernes, c'est le rôle de l'éthique dans ce seuil du troisième millénaire.

\section{LA PERTINENCE DU DROIT POUR L'HOMME MODERNE}

L'importance que le droit joue dans la crise structurelle de la société moderne traité fulcralmente, diffusées et exprime ce descortinamos dans notre pays qui finit par 
générer une chaîne des réactions les plus difficiles est monnaie courante. L'absence de valeurs morales et éthiques, il commence par les dirigeants qui, au lieu de donner l'exemple de douceur et de probité, préfèrent aller le chemin opposé, ancré sous le couvert de l'impunité, qui révolte le citoyen qui paie ses impôts et luttent pour maintenir une vie décente et corriger. Pour un autre changement, certains citoyens horrifiés par cette situation résoudre à agir de la même manière, en vertu de la raison d'être que se l'erreur vient de là-haut n'est aucune raison de ne pas agir de la même manière.

Ce panorama anarchique et le chaos est que les citoyens rencontrent dans leur jour le jour et ne me lasse de demander jusqu'à quand va continuer à regarder tout ce impuissant, sans perspectives de changements, impliqués dans un système juridique qui, avec son ensemble complexe de stériles, les lois ne peut pas créer ces citoyens des mécanismes de défense qui maintient la structure de l'état avec ses lourdes taxes aucun retour de béton. Ce qui est certain, c'est que la possibilité ou la perspective de tout changement dans cet état de choses devrait passer par un examen sérieux et urgent des instituts juridiques et leur application dans la société, qui a laissé à désirer dans la fourniture de compétence.

II y a beaucoup d'écrits sur ce sujet, et la tendance au fil du temps est que plus de juristes et de spécialistes du droit de retour, les yeux sur ce problème, dans le sens d'un changement de direction des directions qui l'a eu le droit, non seulement dans notre société, mais, pourquoi ne pas dire, dans le monde entier, à savoir, l'omission de respecter les intérêts du citoyen qui cherche la justice, au détriment de cette corruption rampante qui fait rage et la disgrâce de la magistrature, précisément en raison de simples intérêts personnels.

Pour le juriste Lenio Streck (2009), le droit ne peut pas combler cette lacune dans notre système juridique, qui perpétue la politique de l'impunité et l'impréparation de l'appareil judiciaire ne pas à répondre aux aspirations du citoyen, non pas par manque de clairvoyance dans le système juridique, sinon à cause de ce qu'il appelle une "crise". Ce que le juriste Gaucho propose un changement de paradigme du modèle juridique actuel déploie dans notre société, qui est très loin de la réalité actuelle dans notre pays. 
C'est exactement cette « crise » détectée par le juriste de diviseur d'eau Rio Grande qui procédera à la conduite de l'avocat dans cette société complexe et mondialisée. Les anciens crimes tellement dégoûtés par la morale de l'homme des cavernes primitif revenir maintenant d'être commis avec une cruauté encore plus grande. Ajoutez à cela d'autres crimes de notre génération, comme les crimes électroniques tels que le clonage de cartes de crédit, les soldes bancaires mouvement criminel par le biais de balances électroniques, envoi d'emails de mendaz avec des liens qui cherchent à capturer les mots de passe à des fins criminelles, entre autres que que l'esprit humain est capable de tisser et exécuter.

Eh bien, c'est dans ce contexte chaotique et encombré que nous avons constaté l'importance du droit à l'homme moderne, comme le droit, dans leur quête de Justice, est le centre d'examen de tous ces maux que sont l'homme moderne. Depuis les drames personnels de droit de la famille avec son état de santé froid, jusqu'à l'immobilité absolue de tout ce que nous pouvons faire face à la tuerie d'innocents aux mains d'un abattu "Punisher" fou et assoiffé de vengeance, le droit devrait être reconsidéré sérieusement pour contempler les maux sociaux qui se dégagent de I'homme moderne en complet désarroi et d'impuissance face aux faits que la présence de tous les jours dans la société.

La pertinence du droit si vous visualiser sur l'involution de morale de la société avec la conduite des gestionnaires publics, qui Excel en rendant le droit un instrument de sa volonté, contre l'intérêt des gouvernés maladroit et incompétent face à ce problème. Le droit doit retentir les intérêts personnels et idéologiques, et si vous jetez dans la recherche de ce changement de scénario que le moment se présente.

L'Empire de la force brute a été ce qui a prévalu dans notre société. En traitant des droits contrefaits les plats de la balance de la Justice jamais osciller pour le citoyen avant l'État, beaucoup moins en faveur des pauvres vers les plus riches, ou même avant les privilégiés contre un anonyme.

Juste pour être une pâle idée de ce qui est dit, les règles de procédure civile établissent des privilèges en faveur de l'entité publique, avec quad pour se défendre et en double, 
et avons-nous mentionné que l'Etat lui-même-juge, en la personne du juge, peut faire appel contre sa propre décision, chaque fois qu'elle est émise contre l'État.

Et pour empirer les choses, les propositions de réforme judiciaires dans notre société ne sont jamais en faveur de l'avocat plaidant de citoyens ; au contraire, toutes les réformes au Brésil s'est retourné contre le justiciable, dans une vaine tentative pour essayer de convaincre la société que la justice est lente et que le retard dans la fourniture de judiciaire examine non seulement de la magistrature elle-même.

Cela remonte à l'heure actuelle que nous vivons, qui est une quête insatiable pour la réhabilitation de l'éthique et la morale dans le monde, de telle sorte qu'une norme minimale de conduite dans les actes de la vie en société et, dans le cas de la Justice, tout ce qui concerne les droits du citoyen. Avocats, juges, procureurs, tous ensemble doivent être des bâtisseurs d'une nouvelle réalité juridique, dans la recherche constante de valeurs éthiques et morales dans la poursuite de la Justice.

Instituts de solution des conflits alternatifs comme nous l'avons déjà mentionné, la médiation, la conciliation et l'arbitrage, sont les bienvenus dans cette reconstruction de processus et la recherche de la Justice, puisqu'elle ne prétend pas à la substitution de la magistrature, mais se présente comme le casting de cette quête de quelque chose de nouveau et que, en fait, remplissent les idéaux de Justice oublié et laissé de côté tout au long de l'histoire.

\section{CONCLUSION}

Avant que tout cela a été dit, le droit doit abandonner ce dogmatique et fermé en supposant pour l'instant et doit marcher vers un pluriel plus. Dans ce monde globalisé, avec tous les brins ont souligné, il n'y a plus de place pour une vision rigide, univoque et très formelle, au contraire, on peut parler maintenant dans la multiplicité des acteurs sociaux (avocats, juges, psychologues, techniciens) et le pluralisme des instituts juridiques qui répond le mieux aux préoccupations que la société moderne, y compris l'arbitrage, de médiation et de conciliation. 
L'heure actuelle conduit au remplacement de l'individualisation du rôle du juge dans un processus de partage des responsabilités des autres acteurs impliqués dans le processus collectif de changements sociaux (les acteurs à l'extérieur de l'appareil judiciaire, comme les pros retromencionados), tous Unis à la recherche d'une solution solide, tangible et concrete pour répondre aux besoins de l'homme actuel. Le pouvoir judiciaire doit promouvoir et faciliter la production des expériences qui favorisent le lien entre lui et la société au lieu de disjonction, de fragmentation et de l'éloignement des citoyens de votre aspiration à la justice.

Dans cette note, la magistrature elle-même devrait encourager les partenariats et harmonisation des pratiques juridiques avec les instituts déjà mentionné que si vous savez par expérience, a été en mesure de donner un retour plus adéquat et satisfaisant pour le citoyen. Ne devrait pas exister ici peur de la concurrence, encore moins la crainte d'apparente des contradictions qui peuvent venir à émerger au milieu de ce processus de changement. Les contradictions doivent être travaillées et pas supprimées, car même si certains d'entre eux peuvent sembler a priori, insurmontable, pourtant ils ne doivent pas rester paralysées ou la fuite et l'histoire des sciences est en construction et déconstruction des paradigmes et surmonter des paradoxes.

Donc, pour tout changement il y a besoin du pouvoir judiciaire d'ouvrir la production des expériences qui favorisent la multiplicité des connaissances et des pratiques juridiques, encourager le partenariat, même entre les perspectives divergentes et théories du courant "status quo ". II faut rechercher le dialogue avec les " mouvements sociaux » et d'autres acteurs que déconstruisent les formes traditionnelles et les burocratizadas de la participation citoyenne dans leur propre histoire.

Aujourd'hui, plus que jamais, il y a un besoin urgent de comprendre que les défis qui se présentent aujourd'hui à l'homme moderne (ou post-moderne) et se rendre compte que toute la production de travaux visant au changement structurel a des implications sociales, ou mieux dire, que toute la production provient de certains changements joue ce rôle juridico-politique et plusieurs relations de pouvoir et de champs de force. 
La marche vers le processus de changement, ouverte sur le sens critique et le potentiel transformatif, dépend de le œuvre de magistrature d'autoévaluation critique sur la signification et les conséquences de leur manière d'agir. II n'est nécessaire pour les pratiques juridiques reposent sur l'interaction avec les besoins sociaux, avec les défis de la mondialisation, toujours faire un contrepoint à la situation actuelle de la magistrature, qui est mis au rebut et aucune condition minimale à répondre aux aspirations des citoyens, face à un fardeau excessif en place il.

Pour cette raison, la justice doit reconnaître et légitimer les autres acteurs qui sont prêts à contribuer à la solution des différends et de satisfaction du citoyen. Au lieu de la question de conflit de juridictions que le pouvoir judiciaire doit agir pour s'asseoir à la table avec les autres professionnels en harmonie avec les besoins vitaux de I'homme, au-delà des problèmes du doigt et les différences notées. Que serait un pas de géant vers un changement de la politique éthique, inducteur et conducteur de la réflexion destiné à non seulement une autocritique, mais également un véritable changement qui vise à mettre le droit sur votre vrai chemin, qui est la poursuite de la Justice dans un monde globalisé, sans oublier l'éthique et la morale et pilastres d'une véritable société démocratique directeur basée sur la primauté du droit.

\section{RÉFÉRENCES BIBLIOGRAPHIQUES}

Adéodat, João Maurício. Déontologie \& rhétorique. 5e édition. São Paulo : Saraiva, 2012.

João Maurício. Légitimation par la procédure juridiquement organisée : notes sur la théorie de Niklas Luhmann. Dans : Éthique et rhétorique : vers une théorie de la dogmatique juridique. p. 53-80, São Paulo : Saraiva, 2002.

BARONI, Robison. Apprêt et l'avocat de l'éthique professionnelle. 3ème ed. LTR Editora Ltda :

Norberto BOBBIO. Positivisme juridique : leçons de la jurisprudence. Traduit par Márcio Pugliesi, Edson Bini, Carlos e. Rodrigues, São Paulo : icône, 1995. 
. Théorie générale de la politique. Traduction de Daniela Beccaccia Versiani, Rio de Janeiro : Campus, 3. Réimpression, 2000.

. Théorie du système juridique. 10. Ed. Traduction de dos Maria Celeste agneau lait Santos, Brasília : Ed. Université de Brasilia, 1997.

Francesco CARNELUTTI. Ce qui a donné naissance à la droite. Traduction fourchette de Ricardo Rodrigues. 1er Ed. Campinas : Editora Russel, 2004.

DWORKIN, Ronald. L'Empire du droit. Martins Fontes : São Paulo, 2007. P. 4

Faria, José Eduardo, droit et mondialisation de l'économie - implications et perspectives, São Paulo : Malheiros, 2010, 1ère édition, 3ème tirage au sort.

FERNANDEZ Jr., introduction à l'étude du droit : technique, de décision, de domination. 3. Ed. São Paulo : Atlas, 2001.

FILS, Willis Santiago guerre. Théorie de la Science juridique. 2eéd. Sami : São Paulo, 2009.

HABERMAS, Jürgen. Droit et démocratie : entre facticité et la validité. 4. Éd. vol. 1. Traduction de Flavius Beno Sienbeneichler, Rio de Janeiro : heure brésilienne, 1997. v 3.

Rudolf Von JHERING. La lutte pour le droit. Martin Claret : São Paulo, 2003.

KELSEN, Hans. Le problème de la Justice. 2. Ed. Traduction de João Baptista Machado, São Paulo : Martins Fontes, 1996.

. Ce qui est Justice-Justice, le droit et la politique dans le miroir de la science. 3ème ed. São Paulo : Martins Fontes, 2001.

. Théorie générale du droit et l'État. 3. Ed. Traduction par Luís Carlos Borges, São Paulo : Martins Fontes, 1998. 
. THÉORIE PURE DU DROIT. Définition de João Baptista Machado. 6 ed. São Paulo : Martins Fontes, 1999, page 1.

NALINI, José Renato. Éthique générale et professionnelle. Rvista des tribunaux : São Paulo, 1997.

Nunes, Pedro. Dictionnaire de l'informatique juridique. 13ème ed. Renouveler : Rio de Janeiro, 1998.

Chaïm Perelman. Éthique et le droit. Martins Fontes : São Paulo, 1996.

REALE, Miguel. Une nouvelle éthique pour le juge. Coord. José Renato Nalini. Magazine des tribunaux : São Paulo, 1994.

SPINOZA, Baruch. L'éthique. Trad. Ahmed Xavier. Ed. Tecnoprint S.a.-Ediouro : São Paulo.

VALLE, Gabriel. Étiva et droit. 1er ed. Editeur de synthèse : São Paulo, 1999.

SETHI, Vincent de Paul. Normes d'éthique professionnelle. Legal éditeur Ltda : forteresse, 1989.

, Eugenio ZAFFARONI Raul STYLE BONDIT. Pouvoir judiciaire, crise, réussites et malentendus. Trad. De Juarez Tavares. Ed. Magazine des tribunaux : São Paulo, 1995. 